\title{
Single Blinded Left-to-Right Comparison Study of Excimer Laser Versus Pulsed Dye Laser for the Treatment of Nail Psoriasis
}

\author{
Nawaf Al-Mutairi · Tarek Noor • Ahmed Al-Haddad
}

To view enhanced content go to www.dermtherapy-open.com

Received: June 12, 2014 / Published online: July 3, 2014

(C) The Author(s) 2014. This article is published with open access at Springerlink.com

\section{ABSTRACT}

Introduction: Nail psoriasis is relatively difficult to treat. Excimer laser has been approved for the treatment of psoriasis since 2000. Pulsed dye laser (PDL) in psoriasis therapy has shown good response rates, with extended remissions. This is the first study assessing both the excimer and PDL lasers in nail psoriasis.

Methods: In a comparison study, excimer laser versus PDL for the treatment of nail psoriasis was evaluated in 42 patients. The right hand nails were treated with excimer laser twice weekly and the left hand nails were treated with PDL once every 4 weeks, for total 12 weeks. The patients were then followed up after a further 12 weeks. Nail Psoriasis Severity Index (NAPSI) scores were recorded at baseline, weeks

Electronic supplementary material The online version of this article (doi:10.1007/s13555-014-0057-y) contains supplementary material, which is available to authorized users.

N. Al-Mutairi $(\bowtie)$

Department of Medicine, Faculty of Medicine, Kuwait University, Kuwait, Kuwait

e-mail: nalmut@usa.net

T. Noor · A. Al-Haddad

Department of Dermatology, Farwaniya Hospital,

Kuwait, Kuwait
4,8 , and 12 , and then at week 24 . Patients were also asked to grade the clinical response to each treatment.

Results: A total of 304 nail changes, 148 with excimer laser and 156 with PDL, were treated. The mean NAPSI score in nails treated with excimer laser was 29.8 at baseline, reduced to 16.3 at week 24. In PDL-treated nails, the NAPSI scores dropped from 29.5 at baseline to 3.2 at week 24 . NAPSI improvement was significantly greater in PDL than excimer $(P=0.001 ; \quad$ Wilcoxon signed-rank test). Thirty-four (81\%) hands achieved NAPSI-50, and $23(55 \%)$ achieved NAPSI-75 at week 12, while complete nail recovery was shown in 6 (14\%) hands treated with PDL. Regarding the hands treated with excimer laser, only 16 (38\%) hands achieved NAPSI-50, while no hands achieved NAPSI-75 at week 12. In general, subungual hyperkeratosis and onycholysis improved significantly, while nail pitting was least responsive. Oil drops and splinter hemorrhages showed moderate response.

Conclusions: When compared to excimer laser, PDL demonstrated a good response for treating nail psoriasis, with minimal side effects. 
Keywords: Dermatology; Excimer laser; Pulsed dye laser; Nail psoriasis; Nail Psoriasis Severity Index

\section{INTRODUCTION}

Psoriasis is a common inflammatory proliferative disease of the skin which affects $1-3 \%$ of the world's population [1]. Nail involvement has long been recognized as a common manifestation of psoriasis. Nail abnormalities are evident in up to $50 \%$ of patients with skin psoriasis and may be the first manifestation of the disease [2]. The lifetime incidence of nail involvement in psoriatic patients is approximated to be between $80 \%$ and $90 \%$ [3]. The disease can be limited to the nails, by affecting a few or several nails.

Nail psoriasis is usually challenging to treat which may be due to the difficulty of topical treatments to penetrate through the nail plate, and the long-term treatment necessary for obtaining any clinical benefit due to the slow growth rate of the nail plate [2]. As proposed by Rich and Scher [4], the Nail Psoriasis Severity Index (NAPSI) has been considered an objective and reproducible tool for assessing nail involvement in psoriatic patients, and can therefore be used to test the effectiveness of therapeutic interventions.

Lasers have been shown to be effective in the treatment of psoriasis vulgaris, but the information for their effectiveness in nails is limited and has just started to emerge. The use of excimer laser for the treatment of psoriasis was first documented in 1997, when it was compared with full body treatments of narrowband ultraviolet (UV) B [5]. The possible explanations for the superior efficacy of the excimer laser over traditional UVB therapy for psoriasis may be due to a higher intensity UV light to plaques, which is more effective in clearing psoriasis [6]. The excimer laser induces T-cell apoptosis characterized by breaks in DNA strands as well as expression of mitochondrial proteins associated with cell death [7].

The pulsed dye laser (PDL), which uses a liquid dye medium rather than a gas medium used by the excimer laser, was first suggested as a treatment for psoriasis in 1992 [8].

The assumption that selective laser ablation of dermal papillary vasculature may lead to resolution of plaque psoriasis is supported by immunohistochemical studies, endorsing changes in the superficial capillary bed in psoriatic lesions, with reduction in the endothelial surface area and proliferation, supplemented by reduction in T-lymphocyte infiltrate $[9,10]$.

Traditional treatments for nail psoriasis, which include topical or intralesional corticosteroids, topical vitamin $\mathrm{D}_{3}$ analogs, photochemotherapy, oral retinoids, methotrexate, and cyclosporin, can be time consuming, painful, or limited by significant toxicities [11]. Treatment of nail psoriasis is disappointing, and a study has shown that only $19.3 \%$ of patients presented with noticeable improvement during any topical treatment [12].

Fingernail disease is noticeable during the entire year and has a negative influence on social, emotional, and business interactions and over $50 \%$ of patients report concomitant pain leading to limitations of daily activities [13].

The aim of this study was to evaluate and compare the effect of PDL versus excimer laser as treatment modalities for nail psoriasis. 


\section{METHODS}

Patients with clinically significant nail changes were selected from psoriasis patients attending the dermatology outpatient clinics in Farwaniya Hospital (Kuwait) after approval from the hospital ethics committee and informed consent signed by each patient. The inclusion criteria were: patients at least 16 years old and psoriatic nail disease refractory to other topical and systemic therapy. Among the individual nail changes looked for were nail pits, discoloration, thickening, onycholysis, longitudinal ridging, subungual hyperkeratosis, splinter hemorrhages, oil drop, and salmon patch. Exclusion criteria were: pregnancy, history of photosensitivity or keloid formation, patient with severe wound around finger nails or paronychia, and systemic therapy for psoriasis within past 6 months. Potassium hydroxide smears were examined in all nails where a possibility of fungal infection was considered. If positive for dermatophytes, these patients were excluded from the study. For each subject, a detailed proforma was completed in which data regarding disease history along with a detailed clinical examination of the nails was recorded. Severity of nail disease was scored at baseline by NAPSI.

It was decided to treat the left hand finger nails with PDL (595 nm, V beam; Candela Laser Corporation, Wayland, MA, USA). The pulsed duration used was $1.5 \mathrm{~ms}$, the beam diameter was $7 \mathrm{~mm}$, and the laser energy was from 8.0 to $10.0 \mathrm{~J} / \mathrm{cm}^{2}$, with $30 \mathrm{~ms}$ cryogen spurt and $30 \mathrm{~ms}$ delay. Treatment was done once every month until nail clearance or for a maximum of 3 months. The right hand finger nails were treated with $\mathrm{XeCl}_{2}$ excimer laser (TALOS; Wave Light Laser Technologie G, Erlangen, Germany). Minimal erythema dose (MED) was calculated on unaffected skin, and treatment was commenced at twice the MED. The fluence was increased by $200 \mathrm{~mJ}$ at each session, until nail clearance had been achieved or for a maximum of 12 weeks (24 treatments). The highest fluence used was $5,000 \mathrm{~mJ} / \mathrm{cm}^{2}$. The patients were reassessed every 4 weeks during the laser treatment, and then 12 weeks after the last laser session, for clinical signs namely pitting, onycholysis, and leukonychia, and for the extent and severity of nail psoriasis on the target fingernail, by means of the NAPSI score. Adverse events were carefully recorded.
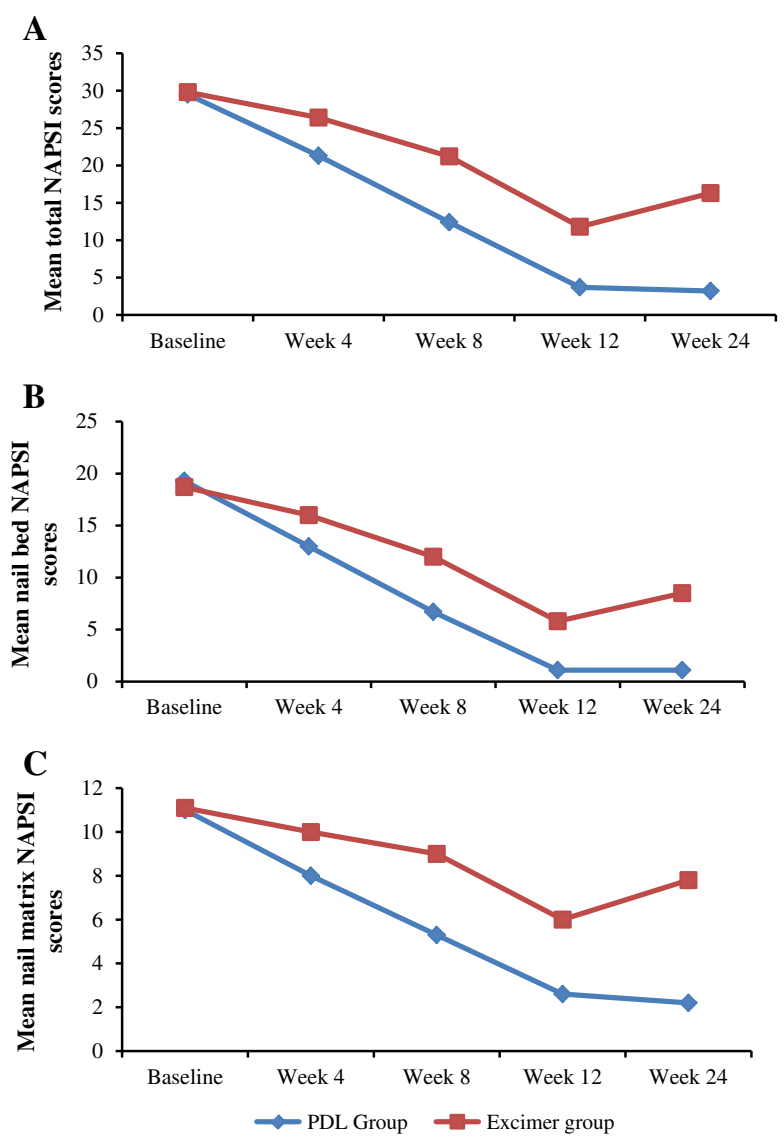

Fig. 1 Serial NAPSI scores in both treatment groups. a Comparison of total nail mean Nail Psoriasis Severity Index (NAPSI) score in PDL and excimer laser groups. b Comparison of nail bed mean NAPSI score in PDL and excimer laser groups. c Comparison of nail matrix mean NAPSI score in PDL and excimer groups 
The Statistical Package for the Social Sciences (SPSS for Windows, Rel. 11.0.1; IBM Corporation, Armonk, NY, USA) was used for the statistical analysis. Analysis of variance for repeated measures and the Friedman test were used to analyze difference of NAPSI measures during the period of treatment. The NAPSI scores were divided into nail matrix, nail bed, and total nail for each nail (Fig. 1).

Patients were asked to grade the clinical response to treatment every 4 weeks during the active treatment period, and then at 12 weeks after the last laser session on a 4-point scale as follows: 0 , no change; 1 , moderately better (up to 50\% overall improvement); 2, much better (between 50\% and $75 \%$ overall improvement); or 3 , cleared (100\% improvement).

\section{RESULTS}

Forty-two patients (23 female and 19 male) aged between 16 and 70 years (mean \pm SD

Table 1 Percentage of nail changes seen in study patients at base line

\begin{tabular}{lll}
\hline Nail changes & $\begin{array}{l}\text { Right hand } \\
(\%)\end{array}$ & $\begin{array}{l}\text { Left hand } \\
\text { (\%) }\end{array}$ \\
\hline Nail matrix psoriasis & & \\
$\quad$ Pitting & 71.4 & 70.9 \\
Discoloration & 38.7 & 39.2 \\
Longitudinal ridging & 32.3 & 31.4 \\
Thickening & 15.3 & 14.9 \\
Nail bed psoriasis & & \\
Onycholysis & 48.3 & 49.1 \\
Subungual & 33.5 & 33.7 \\
hyperkeratosis & & \\
Oil drop discoloration & 5.6 & 5.4 \\
Splinter hemorrhages & 1.7 & 1.6 \\
Salmon patch & 1.6 & 1.6 \\
\hline
\end{tabular}

$40.37 \pm 18.36)$ and affected by symmetrical nail psoriasis in two to five fingernails of both hands (mean $2.14 \pm 0.81$ ) were enrolled. The most common nail change seen in our study was nail pitting (71.1\%) followed by onycholysis (48.7\%; Table 1). A total of 304 nail changes, 148 with excimer laser and 156 with PDL, were treated.

The mean NAPSI scores in nails treated with the excimer laser were 29.8 at baseline, reduced to 11.8 at the third month, and 16.3 at the end of 12 weeks of follow-up after stopping the sessions (Table 2). Statistically significant nail improvement was noted in most patients treated with PDL in the third month as evident from reduction of the mean NAPSI from 29.5 at baseline to 3.7 , in week 12 , and 3.2 at the end of 12 weeks of follow-up $(P<0.001$; Friedman test $)$.

There was no statistically significant difference in NAPSI scores at baseline, but after 3 months treatment period, there were statistically significant difference between the results of excimer laser treatment and PDL, evident by mean NAPSI reduction. The serial NAPSI scores for the two treatment groups at baseline, weeks 4,8 , and 12 , and finally at week

Table 2 Change in nail psoriasis severity index (NAPSI) during the period of treatment, and at the end of follow-up (week 24)

\begin{tabular}{llllll}
\hline Week & \multicolumn{2}{l}{ PDL-treated nails } & & \multicolumn{2}{l}{$\begin{array}{l}\text { Excimer-treated } \\
\text { nails }\end{array}$} \\
\cline { 2 - 3 } \cline { 5 - 6 } & $\begin{array}{l}\text { Mean NAPSI } \\
\text { value }\end{array}$ & SD & & $\begin{array}{l}\text { Mean NAPSI } \\
\text { value }\end{array}$ & SD \\
\hline 0 (baseline) & 29.5 & 18.5 & 29.8 & 17.9 \\
4 & 21.3 & 13.7 & 26.4 & 23.7 \\
8 & 12.4 & 21.4 & 21.2 & 38.5 \\
12 & 3.7 & 17.3 & & 11.8 & 22.6 \\
24 & 3.2 & 18.6 & 16.3 & 10.3 \\
\hline
\end{tabular}

$P D L$ pulsed dye laser, $S D$ standard deviation 
24 follow-up for the whole nail, nail matrix, and nail bed are shown in Fig. 1.

The baseline mean NAPSI score was not significantly different between the PDL and excimer groups (Fig. 1a). The mean decrease in nail bed NAPSI score from baseline to 6 months was significantly greater in the PDL group than in the excimer group (Fig. 1b). The mean decrease in nail matrix NAPSI score from baseline to 6 months was also statistically significant between both the PDL and excimer group (Fig. 1c). The overall evaluation concerning PDL and excimer laser according to difference between the baseline mean NAPSI score and 6 months NAPSI score was statistically significantly different $(P=0.001 ;$ Wilcoxon signed-rank test).

Thirty-four (81\%) hands achieved NAPSI-50, and 23 (55\%) achieved NAPSI-75 at week 12, while complete nail recovery (Fig. 2) was shown in 6 (14\%) hands treated with PDL. Regarding the hands treated with excimer laser, only 16 (38\%) hands achieved NAPSI-50, while no hand achieved NAPSI-75 at week 12.

Regarding the morphology of nail lesions, the most significant improvement was noticed in subungual hyperkeratosis and onycholysis while the least responsive nail change was nail pitting. Oil drops and splinter hemorrhages showed moderate response.

Patient self-evaluation of the clinical response to each treatment at the end of treatment is shown in Table 3. Twenty-five patients in the excimer laser side stated that they did not notice any change, while no one noticed complete clearance. All patients stated that they had improvement in the PDL-treated nail side, with six patients noticing complete cure of their nail psoriasis.

Adverse reactions to excimer laser included slight pigmentation of the treated nail folds in five and a deep brown-black discoloration of the
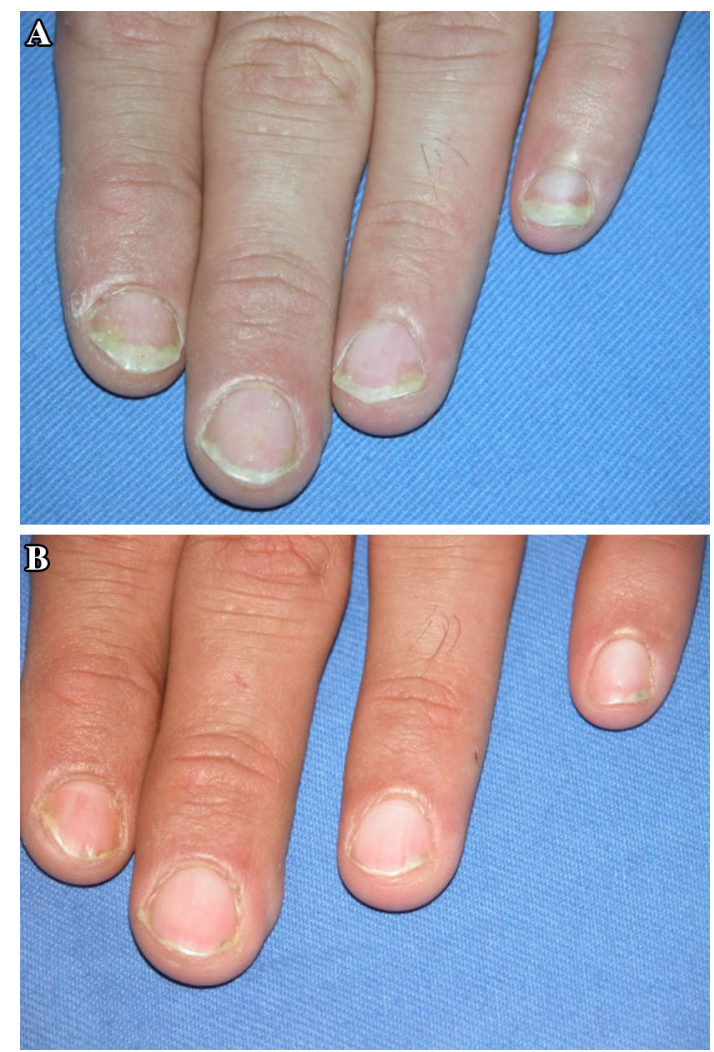

Fig. 2 Effect of pulsed dye laser (PDL) therapy on nail in one of the patient showing almost complete clearance. a Before PDL treatment. b After PDL treatment (week 12)

treated nails in one patient. Adverse reactions to PDL were mild including hyperpigmentation in one patient transient and petechiae in two patients.

\section{DISCUSSION}

To date, there are no studies that compare the efficacy of excimer laser with PDL for the treatment of nail psoriasis. Psoriasis of the nails persists as a problem in patients treated with laser therapy due to the lack of penetration of photons through the nail plate.

The 308-nm excimer laser was approved by the US Food and Drug Administration in 2000 for the treatment of psoriasis. It has now become a well-known treatment modality for 
mild-to-moderate localized psoriasis. Since it is a high-energy monochromatic UVB supplied at $308 \mathrm{~nm}$ that makes it possible to practically treat individual areas of psoriasis in short period of time. In contrast to traditional phototherapy techniques, this handheld device selectively targets lesional skin, thus sparing the surrounding normal skin from unnecessary radiation exposure. It has been shown to be effective in the treatment of different types of psoriasis, such as plaque psoriasis [14, 15], palmoplantar psoriasis [15-18], scalp psoriasis $[15,17,19]$, and inverse psoriasis [20]. The excimer laser appears to exert its therapeutic effect by direct T-cell cytotoxicity. It induces T-cell apoptosis, inhibits cytokine secretion, and impairs antigen presentation by Langerhans cells [16]. However, so far excimer laser has not been found to be effective in limited number of patients of nail psoriasis available in the literature [21]. This could be because of the poor penetration of UVB in the human nail plate [22]. PDL treatment might be considered for the treatment of localized, recalcitrant plaque psoriasis, when other topical therapies have failed [23, 24]. PDL treatment decreases the number of dermal papillary microvessels, which are important pathogenetic targets of psoriasis; PDL therapy, as it selectively targets superficial vessels, is therefore a valid therapeutic approach [25].

In a controlled comparative study of excimer laser and PDL in the treatment of psoriasis,
Taibjee et al. [26] found that both treatments were useful, but the excimer laser was deemed more efficacious than the standard treatment regimen of PDL in the treatment of psoriasis [15].

In this study, we used the right and left hand of each selected patient to create a comparative study about the effect of therapy with PDL and excimer laser in the same patient. According to our results, the PDL was shown to be more effective and less time consuming in the treatment of nail psoriasis than the excimer laser.

In the current study, only 16 (38\%) hands treated with excimer laser showed moderate improvement, and no hand showed complete clearance (according to the patients overall assessment of the results). A pilot study by Aubin et al. [21] for evaluation of the 308-nm monochromatic excimer light delivery system in different chronic localized dermatoses found that four patients with nail psoriasis demonstrated no benefit from this type of treatment.

In contrast, according to the current results, PDL was shown to be a good alternative treatment for nail psoriasis. A marked decrease in NAPSI score was observed with PDL treatments in both nail matrix and nail bed involvement. Thirty-four hands achieved NAPSI-50, and 23 achieved NAPSI-75 at week 12 , while complete nail recovery was shown in 6 hands treated with PDL. The results obtained

Table 3 Patient evaluation of the clinical response to each treatment at the end of treatment period (week 12)

\begin{tabular}{lclcll}
\hline Laser & No change & Moderately better & Much better & Cleared & $\boldsymbol{P}_{\text {value* }}$ \\
\hline Excimer & $25(59.5)$ & $13(31.0)$ & $4(9.5)$ & $0(0.0)$ & $<0.001$ \\
PDL & $0(0.0)$ & $17(40.5)$ & $19(45.2)$ & $6(14.3)$ & \\
\hline
\end{tabular}

Values are given as $n(\%)$

$P D L$ pulsed dye laser

* Paired Wilcoxon signed-rank two-tailed test 
were well maintained throughout the follow-up period of 12 weeks, and NAPSI-50 and NAPSI-75 scores were similar even at the end of week 24 . The personal impression of the patients was good regarding PDL treatment.

Several reports have been published recently about the effect of PDL in the treatment of nail psoriasis. In 2009, Fernández-Guarino et al. [27] evaluated the efficacy of PDL versus photodynamic therapy in the treatment of refractory nail psoriasis in a left-to-right comparative pilot study of 14 patients. Both hands were treated with 595-nm PDL $(7 \mathrm{~mm}$, $6 \mathrm{~ms}, 9 \mathrm{~J} / \mathrm{cm}^{2}$ ), following $3 \mathrm{~h}$ occlusion of methyl-aminolaevulinic acid (MAL) to 1 hand, once a month for 6 months. They showed that both treatments were equally effective in nail bed and nail matrix lesions, and that MAL did not play any role in the treatment of nail psoriasis. In 2010, Oram et al. [28] treated nail psoriasis of 5 patients by 595-nm PDL once monthly for 3 months, with significant improvement, particularly of nail bed lesions. Mean NAPSI score dropped from 21.2 at baseline to 3 after 3 months [28]. In 2012, Treewittayapoom et al. [29] evaluated the effect of two different pulse durations $\left(6 \mathrm{~ms}, 9 \mathrm{~J} / \mathrm{cm}^{2}\right.$ vs. $0.45 \mathrm{~ms}, 6 \mathrm{~J} / \mathrm{cm}^{2}$ both with $7 \mathrm{~mm}$ spot size) of PDL in a left-to-right comparison study of 20 patients. There was no significant difference between the longer and the shorter pulse duration groups. PDL was found to be effective in both nail bed and matrix lesions. A significant reduction in NAPSI scores was observed in both groups after 6 months of the first treatment [29]. Another study evaluating the effect of different pulse durations in the treatment of nail psoriasis with the 595-nm PDL to determine the optimal pulse duration was reported by Goldust and Raghifar [30]. PDL was applied on the proximal and lateral nail folds based on random assignment for forty patients with bilateral fingernail psoriasis. Eighty nails were treated with 6-ms pulse duration and $9 \mathrm{~J} / \mathrm{cm}^{2}$ whereas 80 nails were treated with $0.45-\mathrm{ms}$ pulse duration and $6 \mathrm{~J} /$ $\mathrm{cm}^{2}$. After 6 months of first treatment, there was a significant reduction in overall NAPSI, nail matrix NAPSI, and nail bed NAPSI scores from baseline in both groups; with no significant difference between the two pulse duration groups [30].

A single-blind, intrapatient left-to-right controlled study about the efficacy of PDL plus topical tazarotene versus topical tazarotene alone in psoriatic nail disease was reported by Huang et al. [31]. They included two groups of patients: (1) patients with severe psoriasis who were receiving stable systemic therapy (phototherapy and systemic medication); (2) patients with mild psoriasis who were not receiving systemic therapy. One hand received the experimental treatment (PDL and tazarotene $0.1 \%$ cream) and the other, the control treatment (tazarotene $0.1 \%$ cream). All five fingernails of the experimental hand were treated with 595-nm PDL once a month for 6 months. The mean decrease in modified NAPSI score from baseline to 6 months was significantly more after the experimental treatment than the control treatment. They concluded that PDL plus topical tazarotene $0.1 \%$ cream is an effective and safe therapy in the treatment of nail psoriasis [31].

\section{Limitations}

The study should have been for 6 months of active treatment and then a follow-up for same duration, but because of pressing reasons the authors could not do it. Also, a doubleblinded nature would have carried more weightage. 


\section{CONCLUSION}

In recent years, excimer laser and PDL have demonstrated noticeable effects for the treatment localized plaque psoriasis. Although the excimer laser appears to be, on average, more efficacious than PDL for plaque psoriasis treatment, the contrary seems to be correct for nail psoriasis treatment. Results of the excimer laser in nail psoriasis are poor and time consuming. Whereas, PDL has an excellent response for treating nail psoriasis, with minimal side effects.

\section{ACKNOWLEDGMENTS}

No funding or sponsorship was received for this study or publication of this article. All named authors meet the ICMJE criteria for authorship for this manuscript, take responsibility for the integrity of the work as a whole, and have given final approval to the version to be published. Editorial assistance in the preparation of this manuscript was provided by Dr. Yashpal Manchanda, Farwaniya Hospital, Kuwait.

Conflict of interest. Nawaf Al-Mutairi, Tarek Noor, and Ahmed Al-Haddad declare no conflict of interest.

Compliance with ethics guidelines. This study has received the approval of an institutional review board of Farwaniya Hospital. All procedures followed were in accordance with the ethical standards of the institutional committee on human experimentation and with the Helsinki Declaration of 1975, as revised in 2000 and 2008. Informed consent was obtained from all patients for being included in the study.
Open Access. This article is distributed under the terms of the Creative Commons Attribution Noncommercial License which permits any noncommercial use, distribution, and reproduction in any medium, provided the original author(s) and the source are credited.

\section{REFERENCES}

1. Camp RDR. Psoriasis. In: Champion RH, Burton JL, Burns DA, Breathnach SM, editors. Textbook of dermatology, EDZ, vol. 2. 6th ed. Oxford: Blackwell Science Publications; 1998. p. 1589-649.

2. Cantoresi F, Sorgi P, Arcese A, et al. Improvement of psoriatic onychodystrophy by a water-soluble nail lacquer. J Eur Acad Dermatol Venereol. 2009;23(7):832-4.

3. Samman PD, Fenton DA. The nails in disease. 5th ed. London: Butterworth-Heinemann Ltd.; 1994.

4. Rich P, Scher RK. Nail psoriasis severity index: a useful tool for evaluation of nail psoriasis. J Am Acad Dermatol. 2003;49:206-12.

5. Bonis B, Kemeny L, Dobozy A, Bor Z, Szabo G, Ignacz F. 308 nm UVB Excimer laser for psoriasis. Lancet. 1997;350:1522.

6. Zakarian K, Nguyen A, Letsinger J, Koo J. Excimer laser for psoriasis: a review of theories regarding enhanced efficacy over traditional UVB phototherapy. J Drugs Dermatol. 2007;6:794-8.

7. Novak Z, Bonis B, Baltas E, et al. Xenon chloride ultraviolet $B$ laser is more effective in treating psoriasis and in inducing $\mathrm{T}$ cell apoptosis than narrow-band ultraviolet B. J Photochem Photobiol B Biol. 2002;67:32-8.

8. Hacker SM, Rasmussen JE. The effect of flash lamppulsed dye laser on psoriasis. Arch Dermatol. 1992;128:853-5.

9. Zelickson BD, Mehregan DA, Wedelschfer-Crabb G, et al. Clinical and histological evaluation of psoriatic plaques treated with a flashlamp pulsed dye laser. J Am Acad Dermatol. 1996;35:64-8.

10. Hern S, Allen $\mathrm{MH}$, Sousa $\mathrm{AR}$, et al. Immunohistochemical evaluation of psoriatic plaques following selective photothermolysis of the superficial capillaries. $\mathrm{Br} \mathrm{J}$ Dermatol. 2001;145:45-53. 
11. Gupta AK, Cooper EA. Psoriatic nail disease: quality of life and treatment. J Cutan Med Surg. 2009; 13:S102-6.

12. De Jong EM, Seegers B, Gulink MK, Boezeman JB, Van De Kerkhof P. Psoriasis of the nails associated with disability in a large number of patients: results of a recent interview with 1728 patients. Dermatology. 1996;193:300-3.

13. Rigopoulos D, Gregoriou S, Stratigos A, et al. Evaluation of the efficacy and safety of infliximab on psoriatic nails: an unblinded, nonrandomized, open-label study. Br J Dermatol. 2008;159:453-6.

14. Asawanonda P, Anderson RR, Chang Y, Taylor CR. 308-nm Excimer laser for the treatment of psoriasis: a dose-response study. Arch Dermatol. 2000;136:619-24.

15. Al-Mutairi N, Al-Haddad A. Targeted phototherapy using $308 \mathrm{~nm}$ Xecl monochromatic Excimer laser for psoriasis at difficult to treat sites. Lasers Med Sci. 2013;28:1119-24.

16. He YL, Zhang XY, Dong J, Xu JZ, Wang J. Clinical efficacy of a $308 \mathrm{~nm}$ Excimer laser for treatment of psoriasis vulgaris. Photodermatol Photoimmunol Photomed. 2007;23:238-41.

17. Gattu S, Rashid RM, Wu JJ. 308-nm Excimer laser in psoriasis vulgaris, scalp psoriasis, and palmoplantar psoriasis. J Eur Acad Dermatol Venereol. 2009;23:36-41.

18. Goldberg DJ, Chwalek J, Hussain M. 308-nm Excimer laser treatment of palmoplantar psoriasis. J Cosmet Laser Ther. 2011;13:47-9.

19. Morison WL, Atkinson DF, Werthman L. Effective treatment of scalp psoriasis using the Excimer $(308 \mathrm{~nm})$ laser. Photodermatol Photoimmunol Photomed. 2006;22:181-3.

20. Mafong EA, Friedman PM, Kauvar AN, Bernstein LJ, Alexiades-Armenakas M, Geronemus RG. Treatment of inverse psoriasis with the $308 \mathrm{~nm}$ Excimer laser. Dermatol Surg. 2002;28:530-2.

21. Aubin F, Vigan M, Puzenat E, et al. Evaluation of a novel 308-nm monochromatic Excimer light delivery system in dermatology: a pilot study in different chronic localized dermatoses. $\mathrm{Br} \mathrm{J}$ Dermatol. 2005;152:99-103.
22. Stern DK, Creasey AA, Quijije J, Lebwohl MG. UV-A and UV-B penetration of normal human cadaveric fingernail plate. Arch Dermatol. 2011;147:439-41.

23. Erceg A, Bovenschen HJ, van de Kerkhof PC, Seyger MM. Efficacy of the pulsed dye laser in the treatment of localized recalcitrant plaque psoriasis: a comparative study. Br J Dermatol. 2006;155:110-4.

24. Bovenschen HJ, Erceg A, Van Vlijmen-Willems I, Van De Kerkhof PC, Seyger MM. Pulsed dye laser versus treatment with calcipotriol/betamethasone dipropionate for localized refractory plaque psoriasis: effects on T-cell infiltration, epidermal proliferation and keratinization. J Dermatolog Treat. 2007;18:32-9.

25. Noborio R, Kurokawa M, Kobayashi K, Morita A. Evaluation of the clinical and immunohistological efficacy of the 585-nm pulsed dye laser in the treatment of psoriasis. J Eur Acad Dermatol Venereol. 2009;23:420-4.

26. Taibjee SM, Cheung ST, Laube S, Lanigan SW. Controlled study of Excimer and pulsed dye lasers in the treatment of psoriasis. $\mathrm{Br} \mathrm{J}$ Dermatol. 2005;153:960-6.

27. Fernández-Guarino M, Harto A, Sánchez-Ronco M, García-Morales I, Jaén P. Pulsed dye laser vs. photodynamic therapy in the treatment of refractory nail psoriasis: a comparative pilot study. J Eur Acad Dermatol Venereol. 2009;23:891-5.

28. Oram Y, Karincaoğlu Y, Koyuncu E, Kaharaman F. Pulsed dye laser in the treatment of nail psoriasis. Dermatol Surg. 2010;36:377-81.

29. Treewittayapoom C, Singvahanont P, Chanprapaph $\mathrm{K}$, Haneke E. The effect of different pulse durations in the treatment of nail psoriasis with 595-nm pulsed dye laser: a randomized, double-blind, intrapatient left-to-right study. J Am Acad Dermatol. 2012;66:807-12.

30. Goldust M, Raghifar R. Clinical trial study in the treatment of nail psoriasis with pulsed dye laser. J Cosmet Laser Ther. 2013 (Epub ahead of print).

31. Huang YC, Chou CL, Chiang YY. Efficacy of pulsed dye laser plus topical tazarotene versus topical tazarotene alone in psoriatic nail disease: a singleblind, intrapatient left-to-right controlled study. Lasers Surg Med. 2013;45:102-7. 